\title{
Quantifying the Magnetic Alignment of HIand Dust in the Diffuse ISM
}

\author{
S.E. Clark ${ }^{1}$, J.E.G. Peek ${ }^{2}$, J. Colin Hill ${ }^{1}$, M.E. Putman ${ }^{1}$ \\ ${ }^{1}$ Department of Astronomy, Columbia University \\ New York, NY 10027 \\ ${ }^{2}$ Space Telescope Science Institute \\ Baltimore, MD 21218
}

\begin{abstract}
Sensitive, high resolution observations of Galactic neutral hydrogen (HI) reveal an intricate network of slender linear features, much as sensitive surveys of dust in Galactic molecular clouds reveal ubiquitous filamentary structure. Across the high Galactic latitude sky, diffuse Histructures are aligned with the interstellar magnetic field, as revealed by background starlight polarization (Clark, Peek, \& Putman 2014) and by Planck $353 \mathrm{GHz}$ polarized dust emission (Clark et al. 2015). These discoveries were enabled by the Rolling Hough Transform, a recently developed, open source machine vision algorithm.
\end{abstract}

Keywords. magnetic fields, polarization, ISM: structure

Recent observations reveal that the texture of the interstellar medium (ISM) may be fundamentally filamentary. Elongated linear structures are ubiquitous in molecular clouds (e.g. André et al. 2010), in diffuse dust (e.g. Planck Collaboration 2015a), and in neutral hydrogen (Hı, e.g. McClure-Griffiths et al. 2006). The morphologies of ISM structures probe the physical processes which shaped them. Thus observations motivate a need for quantitative characterizations of ISM filaments. A number of techniques have been used to identify and measure properties of filamentary structures, such as DisPerSE (Sousbie 2011), the Histogram of Relative Orientations (Soler et al. 2013), the Hessian matrix (e.g. Planck Collaboration 2015b), FILFINDER (Koch \& Rosolowsky 2015), and the Rolling Hough Transform (RHT, Clark, Peek, \& Putman 2014).

In particular, techniques for measuring the orientation of filamentary structures invite comparisons between the morphology of ISM filaments and the structure of the ambient magnetic field. The RHT measures intensity as a function of angle in image data, and does not rely on a definition of a filament as a discrete object, thereby allowing high resolution estimates of the orientations of gas structures on the sky. Now that Planck $353 \mathrm{GHz}$ polarized dust emission data are available for the whole sky, the RHT is an ideal tool for large sky area analyses of the relative orientation between ISM structures and the plane-of-sky magnetic field.

We run the RHT on high Galactic latitude data from the GALFA-HIsurvey (Peek et al. 2011) for the region of sky shown in Figure 1. The top panel shows the Hidata, where red, green, and blue represent three different velocity channels. In this velocity-separated image many thin, linear features are apparent. Figure 1 shows the alignment between the orientation of the neutral hydrogen structures as quantified by the RHT and the apparent magnetic field orientation as measured by Planck. See Clark et al. 2015 for details.

The RHT is open source and we support its use. The source code can be found at https:// github.com/seclark, and additions or improvements to the code are welcome.

\section{References}

André, Ph., Men'shchikov, A., Bontemps, S. et al. 2010, Astron. Astrophys., 518, L102

Cabral, B. \& Leedom, L. C., 1993, in Computer Graphics Proc., ACM SIGGRAPH, 263

Clark, S.E., Peek, J.E.G., \& Putman, M.E. 2014, Astrophys. J., 789, 82

Clark, S.E., Hill, J. Colin, Peek, J.E.G., Putman, M.E. et al. 2015, Phys. Rev. Lett., 115, 241302

Heiles, C. 2000, Astron. J., 119, 923

Koch, E. W. \& Rosolowsky, E.W. 2015, Mon. Not. R. Astron. Soc., 452, 3435 

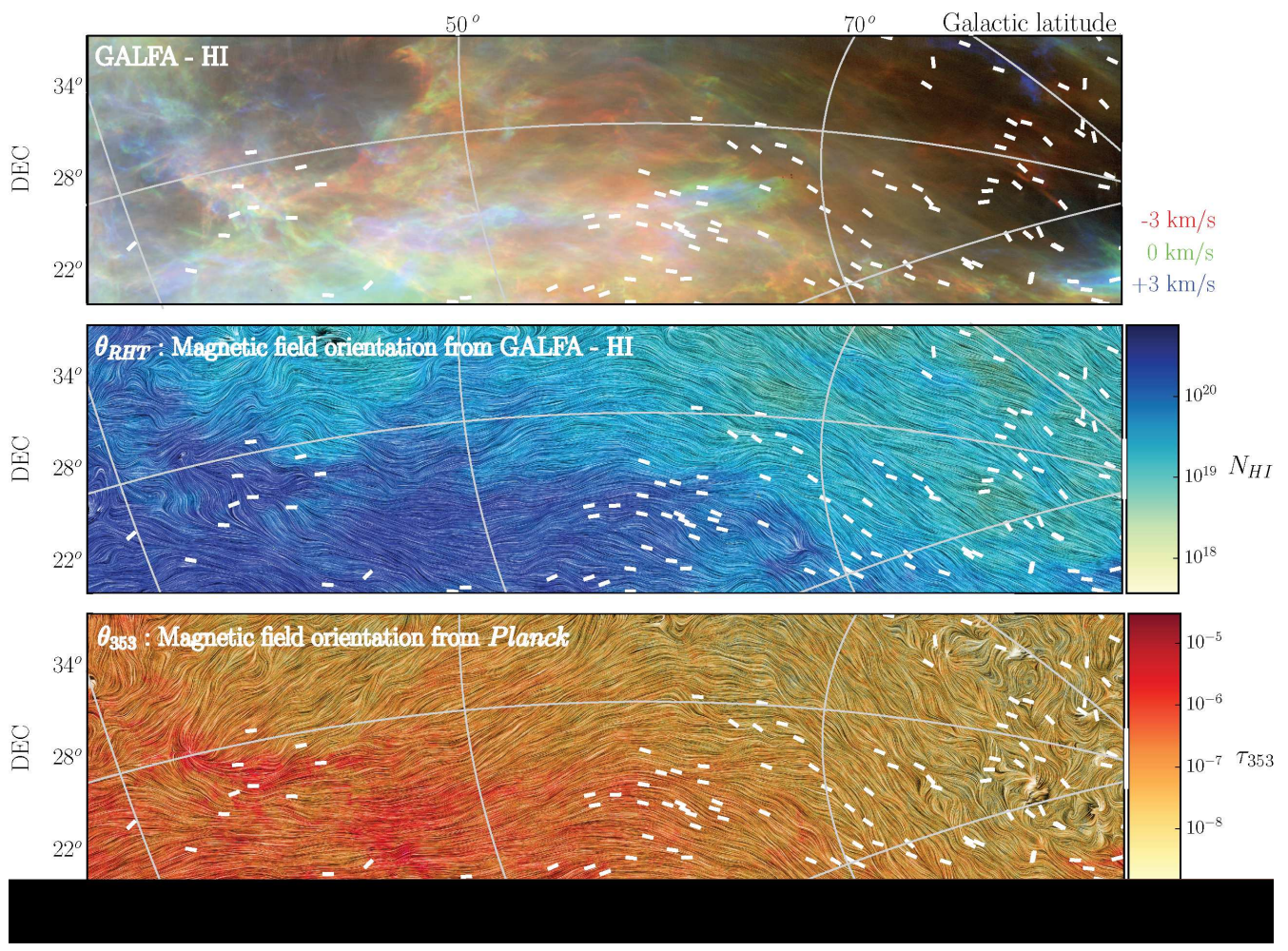

Figure 1. Top panel: GALFA-Hidata binned in $3 \mathrm{~km} / \mathrm{s}$ channels. Red, blue, and green channels represent data centered at $-3 \mathrm{~km} / \mathrm{s}, 0 \mathrm{~km} / \mathrm{s}$, and $+3 \mathrm{~km} / \mathrm{s}$, respectively. Galactic latitude lines are at $30^{\circ}, 50^{\circ}$, and $70^{\circ}$. Galactic longitude lines are at $80^{\circ}, 50^{\circ}$, and $20^{\circ}$, from top right to bottom right. Thin linear structures abound, particularly at high Galactic latitude. Bottom two panels: colors represent Hicolumn density (middle) and integrated line-of-sight opacity (bottom). The swirls of light and dark lines in the two lower panels represent the implied magnetic field orientation. The middle panel shows the orientation of linear Hifeatures obtained with the RHT. The bottom panel shows the implied magnetic field orientation from the Planck $353 \mathrm{GHz}$ polarization data. The Stokes $Q$ and $U$ fields used to generate the angles were Gaussian smoothed with a beam of FWHM $=1^{\circ}$. This texture is generated using Line Integral Convolution (LIC; Cabral \& Leedom 1993), the convolution of noise with a vector field, and visualized as the image lightness. White pseudovectors represent measurements of optical starlight polarization angles (Heiles 2000). The Planck data are noise-dominated at high Galactic latitudes, as visually evident in the LIC pattern. The Arecibo HIdata is sensitive at high latitudes, and the LIC pattern appears nicely combed. Bottom two panels are published in Clark et al. 2015.

McClure-Griffiths, N. M., Dickey, J. M., Gaensler, B. M., et al. 2006, Astrophys. J., 652, 1339 Peek, J. E. G., Heiles, C., Douglas, K. A., et al. 2011, Astrophys. J. Suppl., 194, 20

Planck Collaboration 2015, Astron. Astrophys. 576, A104

Planck Collaboration, Astron. Astrophys., submitted, arXiv:1409.6728

Soler, J. D., Hennebelle, P., Martin, P. G., et al. 2013, Astrophys. J., 774, 128

Sousbie, T. 2011, Mon. Not. R. Astron. Soc., 414, 350 